
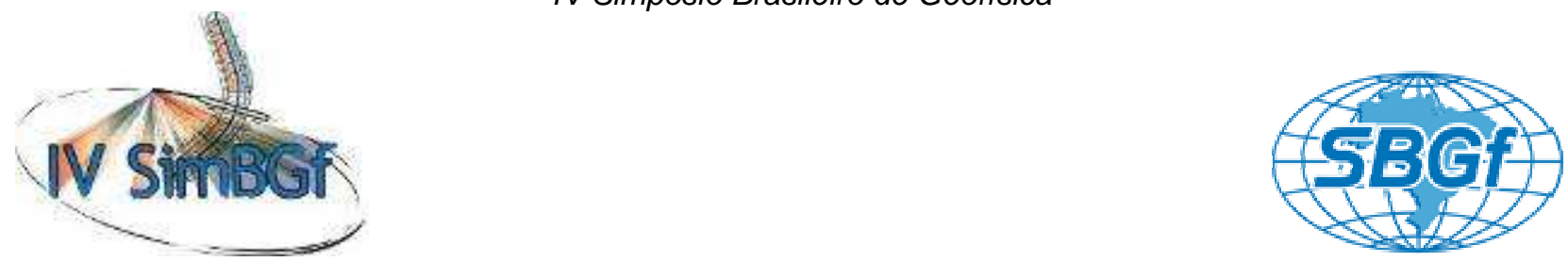

\title{
Petrofísica de Laboratório - Estado da Arte e Perspectivas
}

Rodrigo Surmas, André Luiz Martins Compan, Dario Abilio Cruz, Heitor Vitor Ribeiro Camargo, Rodrigo Skinner, Vinícius de França Machado, Petrobras/CENPES/PDP/TRA (Tecnologia de Recuperação e Análise de Reservatórios)

Copyright 2010, SBGf - Sociedade Brasileira de Geofísica

Este texto foi preparado para a apresentação no IV Simpósio Brasileiro de Geofísica, Brasília, 14 a 17 de novembro de 2010. Seu conteúdo foi revisado pelo Comitê
Técnico do IV SimBGt, mas não necessariamente representa a opinião da SBGf ou de seus associados. É proibida a reprodução total ou parcial deste material para propósitos comerciais sem prévia autorização da SBGf.

\section{Resumo}

Na engenharia de reservatórios de petróleo, a Petrofísica é a responsável pela caracterização dos fenômenos físicos e químicos relacionados à estrutura porosa e sua interação com os fluidos presentes. Participa da avaliação do potencial de exploração e explotação das jazidas fornecendo parâmetros para a determinação da sua capacidade de armazenamento e de produção.

A Petrofísica de Laboratório, em especial, tem papel muito importante nesta caracterização. Através de ensaios realizados diretamente em amostras de rochas de um reservatório podem ser obtidos dados tanto para a construção do modelo geológico quanto para a simulação numérica do fluxo de fluidos.

Neste trabalho os principais processos da Petrofísica de Laboratório são apresentados, bem como discussões sobre suas principais limitações e perspectivas futuras, dentre elas as iniciativas de Petrofísica Computacional, quando as amostras de rocha têm sua estrutura de poros e matriz digitalizada e a elas são aplicados modelos numéricos para simulação dos ensaios.

\section{Introdução}

O conhecimento das propriedades geológicas e de fluxo de rochas-reservatório é de fundamental importância para a engenharia de petróleo. Estes dados são obtidos de diversas fontes diferentes: dados sísmicos, caracterização geológica, testes de formação, perfilagem e análise de testemunhos em laboratório.

A Petrofísica é a responsável pela caracterização dos fenômenos físicos e químicos relacionados à estrutura porosa e sua interação com os fluidos presentes. Participa da avaliação do potencial de exploração e explotação das jazidas fornecendo parâmetros para a determinação da sua capacidade de armazenamento e de produção.

O trabalho da Petrofísica de Laboratório é iniciado na obtenção de amostras de rocha representativas do reservatório, processo que é delicado e custoso, exigindo cuidados especiais desde o planejamento da perfuração do poço, passando pela correto corte das amostras e terminando com o seu correto acondicionamento e conservação.

Na primeira fase é realizada a Petrofísica de Rotina das amostras obtidas. Esta etapa é importante na caracterização do testemunho como um todo, sendo uma importante ferramenta para a construção do modelo geológico do reservatório e servindo como base para a escolha de amostras a serem utilizadas na Petrofísica Especial.

Na Petrofísica Especial os ensaios são mais custosos e demorados, mas os resultados são muito mais detalhados. Sua importância é vital tanto para a construção do modelo geológico quanto para a simulação numérica do fluxo. Neste trabalho os principais ensaios de Petrofísica de Rotina e Especial serão discutidos.

Desenvolvimentos recentes na área de microtomografia computadorizada tornaram possível a representação digital de meios porosos reais em alta resolução. Aliado a algoritmos desenvolvimentos com o objetivo de simular os processos físicos ocorrendo nestes meios, pode-se vislumbrar a perspectiva de complementar os ensaios laboratoriais com resultados obtidos numericamente por estes modelos. Na última parte deste trabalho estes desenvolvimentos serão apresentados.

\section{Obtenção de Amostras}

As amostras petrofísicas são obtidas na operação de testemunhagem e na coleta de amostras laterais.

Os testemunhos são obtidos concomitantemente a perfuração de um poço pela substituição da broca regular por um conjunto formado por uma broca especial vazada (coroa) e um barrilete, este responsável por acomodar a rocha cortada. Esta operação é especialmente custosa pelos seguintes motivos: (i) envolve uma série de manobras da coluna de perfuração uma vez que o barrilete acomoda em média 20 metros de rocha; (ii) a velocidade de avanço da perfuração é reduzida, (iii) aumenta os riscos de prisão da coluna; Desta forma a testemunhagem é feita em parte dos poços perfurados, sendo especialmente indicados nas etapas iniciais de exploração e desenvolvimento.

A coleta de amostras laterais é realizada em poços já perfurados por ferramenta a cabo. Consiste num amostrador que desce ao reservatório e na profundidade indicada se aproxima da parede do poço para cortar um pequeno pedaço de rocha. Tais amostradores possuem boa capacidade e numa mesma corrida coletam razoável quantidade de amostras em profundidades diferentes. 
A coleta de amostras laterais é menos custosa e mais rápida que a testemunhagem, mas seus resultados não são exatamente comparáveis. Enquanto a primeira recupera pequenos cilindros de comprimento 2 a $3 \mathrm{~cm}$ e 1" de diâmetro, a segunda um cilindro de comprimento continuo e 4" de diâmetro, chamado de testemunho.

O testemunho contínuo, Figura 1, permite avaliações geológicas bem amplas sobre as características das rochas e seu histórico de deposição, bem como uma grande gama de ensaios petrofísicos realizados nos plugues retirados dele. As amostras laterais, em contrapartida, são limitadas pelo seu pequeno tamanho, que dificulta ou impede a sua utilização em muitos ensaios petrofísicos.

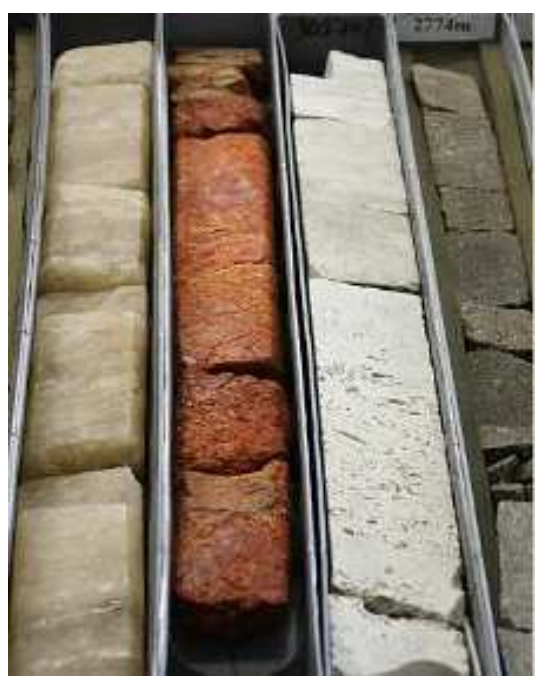

Figura 1 - Foto de testemunho

\section{Testes de Petrofísica de Laboratório}

Quando o testemunho chega ao laboratório (em especial, no CENPES), uma tomografia completa a uma resolução próxima de $0,5 \mathrm{~mm}$ por pixel é realizada em todo seu comprimento. Os objetivos desta tomografia são avaliar os possíveis danos na operação de testemunhagem e auxiliar no processo de caracterização da rocha a partir das imagens da sua estrutura de matriz e poros para avaliações qualitativas e quantitativas.

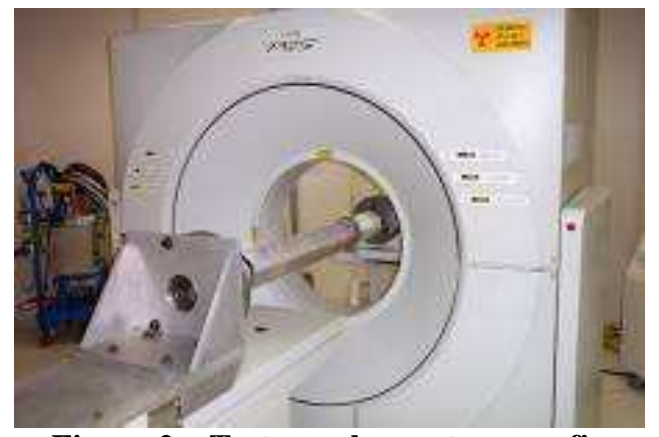

Figura 2 - Testemunho em tomografia
Dos testemunhos são retirados plugues (pequenos cilindros de cerca de $5 \mathrm{~cm}$ de comprimento e 1,5" de diâmetro) afastados de 20 a $30 \mathrm{~cm}$ uns dos outros. Nestes plugues ou nas amostras laterais são analisadas as propriedades de permeabilidade absoluta, porosidade e peso específico da matriz de rocha. Além disso, são realizadas as lâminas delgadas para estudos petrográficos e retirados fragmentos para análise mineralógica (microscópio de varredura - MEV e difratometria de raios- $x$ - DRX).

Estas são chamadas de análises de rotina. São realizadas em todos os plugues ou amostras laterais coletadas, tem especial importância nas etapas exploratórias e por isto mesmo devem ter seus resultados disponibilizados a tempo de serem consideradas para tomada de decisão de testes de formação, completação ou abandono de poço.

Ensaios de Rotina:

\begin{tabular}{|c|l|}
\hline $\begin{array}{c}\text { Tipo de Ensaio } \\
\text { Absoluta }\end{array}$ & \multicolumn{1}{|c|}{ Principal utilização } \\
\hline $\begin{array}{c}\text { Porosidade } \\
\text { Efetiva }\end{array}$ & Capacidade de armazenamento. \\
\hline $\begin{array}{c}\text { Peso Específico } \\
\text { de Matriz de } \\
\text { Rocha }\end{array}$ & $\begin{array}{l}\text { Avaliação média dos minerais que } \\
\text { compõem a rocha. }\end{array}$ \\
\hline Petrografia & $\begin{array}{l}\text { Identificação de mineralogia, } \\
\text { diagênese, teor de argilas. }\end{array}$ \\
\hline DRX e MEV & $\begin{array}{l}\text { Avaliação quantitativa dos tipos de } \\
\text { minerais. }\end{array}$ \\
\hline $\begin{array}{c}\text { Tomografia } \\
\text { computadorizada }\end{array}$ & $\begin{array}{l}\text { Imageamento da estrutura de } \\
\text { matriz e poros para modelagem } \\
\text { computacional de outros } \\
\text { parâmetros petrofísicos. }\end{array}$ \\
\hline
\end{tabular}

\section{Tabela 1 - Análises de Rotina}

Na seqüência da Petrofísica de Rotina são realizados os trabalhos da Petrofísica Especial. Estes são normalmente mais sofisticados e demorados, sendo realizados apenas em uma parte do material coletado.

Seus principais parâmetros são as propriedades capilares e viscosas que se desenvolvem na interação de rochas e fluidos e determinam as características de equilíbrio estático (Swi) e escoamento multifásico (curvas de pressão capilar e permeabilidade relativa); as propriedades elétricas (para calibração de perfil resistividade); as propriedades de resistência e elasticidade mecânicas; propriedades de ressonância magnética nuclear (para calibração do perfil RMN); propriedades das ondas acústicas (utilizadas pela interpretação sísmica); propriedade de afinidade da rocha ao fluido (índice de molhabilidade). Há ainda testes especificamente desenhados para avaliação de danos ao reservatório por interação rocha-fluido e para avaliação de métodos de recuperação avançada de petróleo (Enhanced Oil Recovery - EOR), tais como, injeção de vapor, injeção polímeros, injeção de gases miscíveis, entre outros. 


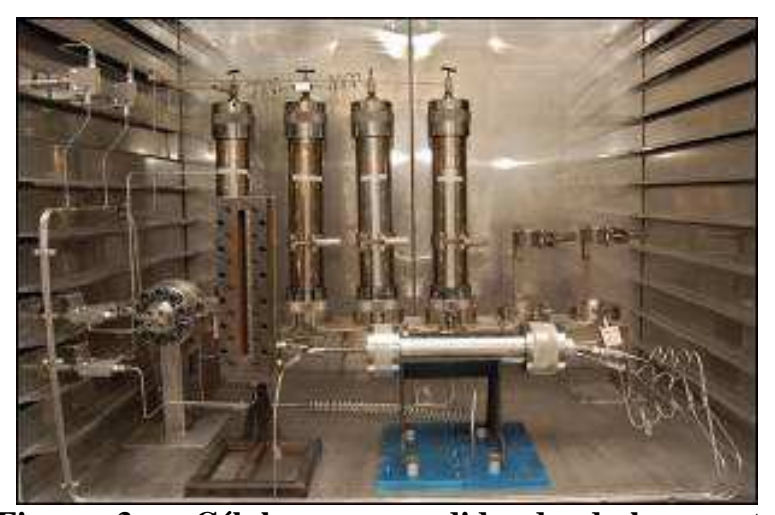

Figura 3 - Célula para medida de deslocamento multifásico em pressão e temperatura de reservatório.

\begin{tabular}{|c|c|}
\hline Tipo de Ensaio & Principal utilização \\
\hline $\begin{array}{l}\text { Escoamento } \\
\text { multifásico }\end{array}$ & $\begin{array}{l}\text { Curvas de permeabilidade relativa e } \\
\text { pontos de saturação finais. }\end{array}$ \\
\hline Capilaridade & $\begin{array}{l}\text { Curvas de pressão capilar em } \\
\text { função da saturação de fluidos. }\end{array}$ \\
\hline Molhabilidade & $\begin{array}{l}\text { Ângulo de contato e índices de } \\
\text { molhabilidade. }\end{array}$ \\
\hline $\begin{array}{l}\text { Propriedades } \\
\text { elétricas }\end{array}$ & $\begin{array}{l}\text { Expoentes de cimentação (m) e de } \\
\text { saturação }(n) \text { da Lei de Archie. }\end{array}$ \\
\hline $\begin{array}{c}\text { Propriedades de } \\
\text { RMN }\end{array}$ & $\begin{array}{l}\text { Porosidade, parâmetros de corte de } \\
\text { T2 e modelos de permeabilidade. }\end{array}$ \\
\hline $\begin{array}{l}\text { Propriedades } \\
\text { Mecânicas }\end{array}$ & $\begin{array}{l}\text { Sensibilidade da permeabilidade e } \\
\text { da porosidade ao estado de } \\
\text { tensões, módulos de Young e } \\
\text { Poisson, ângulo de atrito e coesão, } \\
\text { resistência a compressão e a } \\
\text { tração. }\end{array}$ \\
\hline $\begin{array}{l}\text { Propriedades } \\
\text { Acústicas }\end{array}$ & $\begin{array}{l}\text { Velocidades de ondas } \\
\text { compressionais e cisalhantes. }\end{array}$ \\
\hline $\begin{array}{c}\text { Dano a Formação } \\
\text { e Recuperação } \\
\text { Avançada de } \\
\text { Petróleo }\end{array}$ & $\begin{array}{l}\text { Estudos de injetividade, métodos } \\
\text { de recuperação secundária e } \\
\text { terciária, saturações residuais, } \\
\text { eficiência de tratamentos, estudos } \\
\text { de dano e estimulação, interação } \\
\text { rocha-cimento, testes de } \\
\text { acidificação, testes de inibidores, } \\
\text { WAG, simuladores de fraturamento, } \\
\text { testes de brocas. }\end{array}$ \\
\hline
\end{tabular}

\section{Tabela 2 - Análises Especiais}

\section{Incertezas e Integração de Dados de Laboratório}

Em função da sensibilidade das propriedades petrofísicas obtidas no laboratório, uma série de cuidados devem ser tomados no manuseio de amostras, planejamento e realização testes para que as propriedades obtidas sejam representativas das rochas em condição de reservatório. Limitações e erros experimentais podem trazer incertezas importantes nestas análises.

Durante o processo de testemunhagem, por exemplo, há riscos de danos pela invasão de fluido de perfuração e alterações pela expansão dos fluidos e da rocha. Durante o transporte da rocha podem haver danos mecânicos decorrentes do manuseio.
De maneira geral, as propriedades petrofísicas são influenciadas pela pressão e temperatura do reservatório, devendo estas ser reproduzidas em laboratório. Mais do que isso, os técnicos responsáveis pelo projeto e execução dos ensaios devem conhecer e prever os fenômenos físicos e químicos que se desenvolvem para adequar as condições de contorno dos testes (dadas as limitações dos laboratórios) às condições reais dos reservatórios.

Deve-se considerar ainda que os ensaios de Petrofísica são realizados em pequenos plugues não representativos do reservatório como um todo. Desta maneira os processos de modelagem geológica e de fluxo passam pelo ajuste e calibração de outras ferramentas de princípios e escalas diversas, processo chamado de integração rocha-perfil-teste-sísmica (Figura 4).

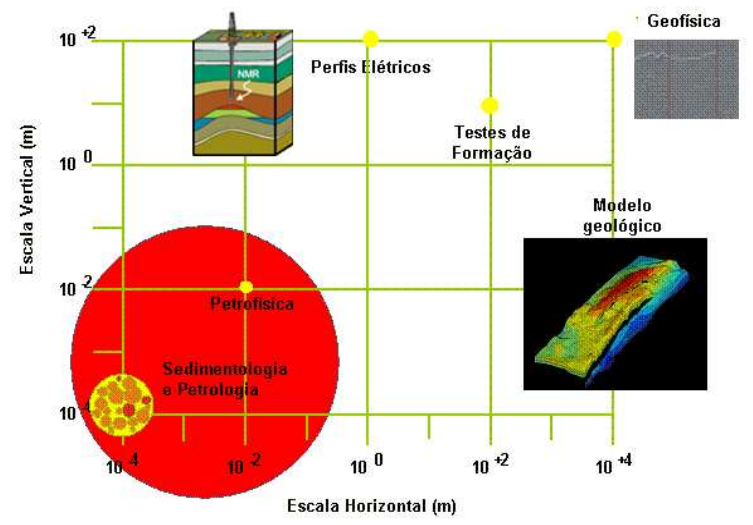

Figura 4 - Integração de ferramentas

\section{Petrofísica Computacional}

Dentre os mais recentes testes realizados em testemunhos e plugues encontra-se a microtomografia computadorizada. Este ensaio consiste na medição do coeficiente de atenuação da rocha em alta resolução, com o objetivo de extrair informações sobre a estrutura e composição desta rocha, bem como sobre a distribuição de fluidos dentro dela, em uma escala variando desde milímetros até nanômetros.

Recentemente esta técnica tem se tornado mais confiável devido ao uso de lentes especiais, o melhoramento e aceleração dos algoritmos de reconstrução e o melhoramento dos filtros dos feixes. Estes melhoramentos têm contribuído também para eliminar a necessidade de corte da rocha em muitos casos, tornando este teste cada vez menos destrutivo.

Como resultado técnico tem-se observado uma melhora substancial no contraste e na resolução das imagens obtidas, além da aceleração de obtenção destas imagens.

O resultado da microtomografia frequentemente é uma série de imagens seqüenciais como a figura 5 . 


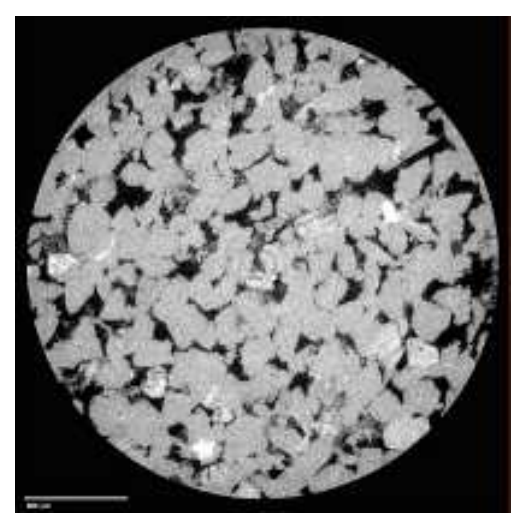

Figura 5 - Exemplo de imagem microtomografada

As partes mais claras desta imagem estão relacionadas a coeficientes de atenuação mais altos, característicos de materiais de densidade mais alta. As partes mais escuras podem ser relacionadas aos vazios deste meio poroso.

É possível, através deste método e de uma série de medições auxiliares como, por exemplo, imagens de MEV e de lâmina, imagens microtomográficas tomadas em energias de feixe diferentes e imagens da amostra saturada com diferentes fluidos, determinar a litologia e mineralogia da rocha, teor de argilas, distribuição de fluidos dentro dela em estados de saturação diversos e sua macro e microporosidades.

Como exemplo de aplicação destas técnicas, na figura 6 é apresentada a distribuição de saturação de um determinado fluido em uma rocha em um processo de invasão imiscível obtido através do imageamento da amostra seca, saturada completamente com o fluido deslocante, saturada completamente com o fluido deslocado e saturada com uma proporção qualquer de fluido deslocante e deslocado. Reconhecendo que o coeficiente de atenuação é proporcional a densidade de um determinado material, pode-se determinar a saturação dos fluidos em uma condição qualquer durante este processo de invasão acompanhando-se este processo por um tomógrafo.

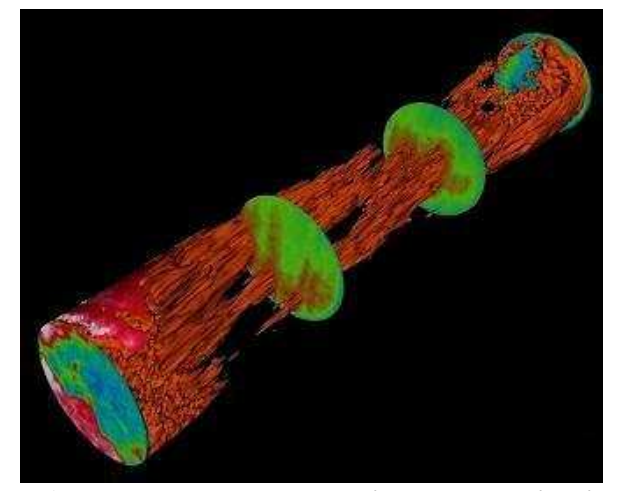

Figura 6 - Exemplo de determinação da distribuição de determinado fluido em uma rocha
Se a resolução da microtomografia for suficiente para representar com fidelidade os principais poros desta rocha, pode-se obter um modelo do meio poroso microtomografado em três dimensões (figura7).

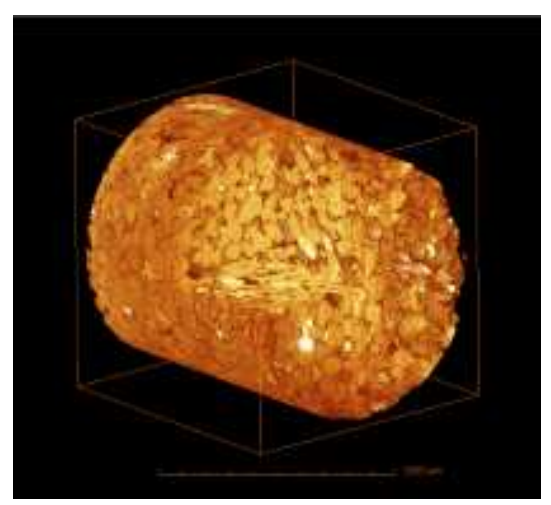

Figura 7 - Composição de imagens estabelecendo um meio poroso tridimensional

Esta representação pode ser utilizada na simulação numérica de diversos fenômenos físicos importantes que podem ocorrer na rocha. Dentre as propriedades petrofísicas que podem ser calculadas nestas simulações estão a porosidade, a distribuição de tamanho de poros, a permeabilidade absoluta e relativa, a pressão capilar, as propriedades acústicas da rocha (velocidade do som das ondas $S$ e $P$ ), parâmetros elétricos (coeficientes de cimentação e saturação) e parâmetros magnéticos (RMN).

Estas propriedades tem-se calculado de forma cada vez mais exata devido ao emprego de algoritmos cada vez mais robustos, utilizando processamento paralelo de alto desempenho e à validação mais extensa destes algoritmos para vários tipos de materiais. Novos algoritmos visando a determinação de outras propriedades também tem sido empregados. Todos estes avanços tem aumentado a confiabilidade das propriedades assim calculadas e expandido a aplicação deste método.

\section{Conclusões}

Neste trabalho foram apresentados os principais processos envolvidos na determinação de propriedades petrofísicas em laboratório. Todos estes processos, da obtenção das amostras dos reservatórios, transporte e seu armazenamento até as técnicas de laboratório e condições experimentais utilizadas necessitam de um cuidadoso planejamento para a obtenção de resultados com um mínimo de incertezas. Foi apresentada também a área de Petrofísica Computacional, candidata a complementar os dados obtidos em laboratório de maneira realista, de forma a aumentar a confiabilidade dos resultados obtidos, testar sua sensibilidade e ampliar sua aplicação para amostras cujos ensaios experimentais são complicados ou até mesmo impossíveis. 


\section{Referências}

Arns, C.H. et al. (4 co-autores), dezembro de 2005. Porescale characterization of carbonates using x-ray microtomography. Society of Petroleum Engineers (SPE), v. $10: 475-484$.

Ottesen, R. \& Hjelmeland, O., 2008. The value added from proper core analysis. Proceedings do International Symposium of the Society of Core Analysts, Abu Dhabi, Emirados Arabes, n. 2008-04.

Tomutsa, L et al. (4 co-autores), janeiro de 2001. Imaging techniques applied to the study of fluids in porous media. National Institute for Petroleum and Energy Research, Bartlesville, Estados Unidos, n. 485.

Torsaeter, O. \& Abtahi, M., janeiro de 2003. Experimental reservoir engineering - laboratory workbook. Norwegian University of Science and Technology. 